den, weil die Fortsetzung in $l$-Richtung nicht eindeutig ist.

Wenn man sich auf den Standpunkt stellt, daß der fünften Dimension eine tiefere Bedeutung nicht zukommt, so enthält unsere Gl. (22) genau den physikalischen Kern des fünfdimensionalen Erhaltungssatzes.
Es kann natürlich im Rahmen der hier vorliegenden Behandlung auf dem Boden der klassischen Feldtheorie nicht ausgeschlossen werden, daß die quantisierte Theorie eine neue Interpretation dieses fünfdimensionalen Erhaltungssatzes liefert.

Herrn Dr. Strauss danke ich für die Anregung zu dieser Arbeit.

\title{
Schwankungen der primären kosmischen Strahlung
}

\author{
Von E. WaibeL * \\ Aus dem Max-Planck-Institut für Aeronomie, Lindau/Han. \\ (Z. Naturforschg. 17 a, 135--142 [1962] ; eingegangen am 23. Dezember 1961)
}

\begin{abstract}
Am 23. 8. 1960 wurden über Lindau (Norddeutschland) bei relativ ruhiger kosmischer Strahlung mit einer Ballonsonde die Häufigkeiten der primären $\alpha$-Teilchen, Protonen und der Gesamtstrahlung gemessen (magnetische Mindeststeifigkeit 2,38 GV). Eine Extrapolation der Strahlungsintensitäten auf $0 \mathrm{~g} / \mathrm{cm}^{2}$ führt für die Gesamtstrahlung zu $0,134 \pm 0,003\left(\mathrm{~cm}^{2} \mathrm{sec} \mathrm{sr}\right)^{-1}$ und für die $\alpha$-Teilchen unter Berücksichtigung der Aufspaltung schwerer Kerne zu 0,0175 $\pm 0,0006\left(\mathrm{~cm}^{2} \mathrm{sec} \mathrm{sr}\right)^{-1}$. Ein Vergleich mit in der Zeit von 1957-1959 gemachten Messungen, z. Tl. bei Forbush-Effekten, führt sowohl für die Gesamtstrahlung als auch die $\alpha$-Komponente zu dem gleichen logarithmischen Schwankungs. faktor von 2,1 $\pm 0,3$, bezogen auf die Neutronenregistrierung am Boden in Lindau. Die Häufigkeit der extrapolierten $\alpha$-Teilchen macht im Mittel $13 \%$ der Gesamtstrahlung aus.
\end{abstract}

Während der Phase hoher und höchster Aktivität im 11-jährigen Sonnenfleckenzyklus wurde in der Zeit von April 1957 bis August 1960 in einer Serie von Ballonaufstiegen das Ionisationsspektrum der kosmischen Strahlung gemessen. Zwei dieser Experimente fanden während starker ForbusH-Effekte statt. Hierbei wurden sowohl die absoluten Intensitäten der Gesamtstrahlung, der Protonen und der a-Teilchen ermittelt als auch die zeitlichen Schwankungen dieser Komponenten untersucht. Die Trennung der Teilchen verschiedener Ladung wurde durch die lineare Abhängigkeit der spezifischen Ionisation vom Quadrat der Ladungszahl ermöglicht bei Ausschluß langsamer, stark ionisierender Teilchen.

Die $\alpha$-Teilchen-Komponente der kosmischen Strahlung zeichnet sich durch ihre weitgehende Freiheit von Sekundäreffekten, wie z. B. der Albedo und ihre doch relativ hohe Teilchenintensität vor den übrigen primären Komponenten mit höherer oder niedrigerer Ladungszahl aus. Dies ist von großer Bedeutung, wenn man absolute Intensitäten vergleichen will, die mit verschiedenen Methoden ermittelt wurden.

Im Zusammenhang mit den möglichen Modulationsmechanismen ist andererseits das Verhältnis

\footnotetext{
* Jetzt Physikalisch-Technische Bundesanstalt Braunschweig.
}

der zeitlichen Schwankungen der Komponenten zueinander von Interesse; das gilt insbesondere für die primären Protonen und $\alpha$-Teilchen. Soweit die Experimente mit einheitlichen Geräten erfolgen, entfallen etliche systematische Fehler, die die absoluten Häufigkeiten beeinflussen. Dies trifft für die Reihe unserer Messungen zu, so daß die Schwankungen der untersuchten Komponenten zueinander und in bezug auf die Neutronenregistrierungen am Erdboden aus Relativwerten gewonnen werden können.

Über den letzten Ballonaufstieg dieser Serie am 23. 8. 60 wird in dieser Arbeit ausführlich berichtet. Die Strahlung war an diesem Tage nicht durch einen Forbush-Effekt gestört, so daß die Messungen zum Vergleich mit den stark verminderten Strahlungswerten, besonders am 16. 7. 59, sehr geeignet sind.

\section{Die experimentelle Technik}

Das Aufstiegsgerät besteht aus drei zu einem Teleskop zusammengefaßten Proportionalzählern zur Messung der spezifischen Ionisation der schnellen Partikel, der zugehörigen Elektronik und aus einem kleinen Teleskop aus Geiger-MülLER-Zählern zur Registrierung der weichen Strahlung.

Da die Funktionsweise der Sonde bereits eingehend in Anm. ${ }^{1}$ diskutiert wurde bis auf einige Zusätze, sei

1 E. Waibel, Mitt. Max-Planck-Inst. Aeronomie Nr. 1 (Berlin 1959). 


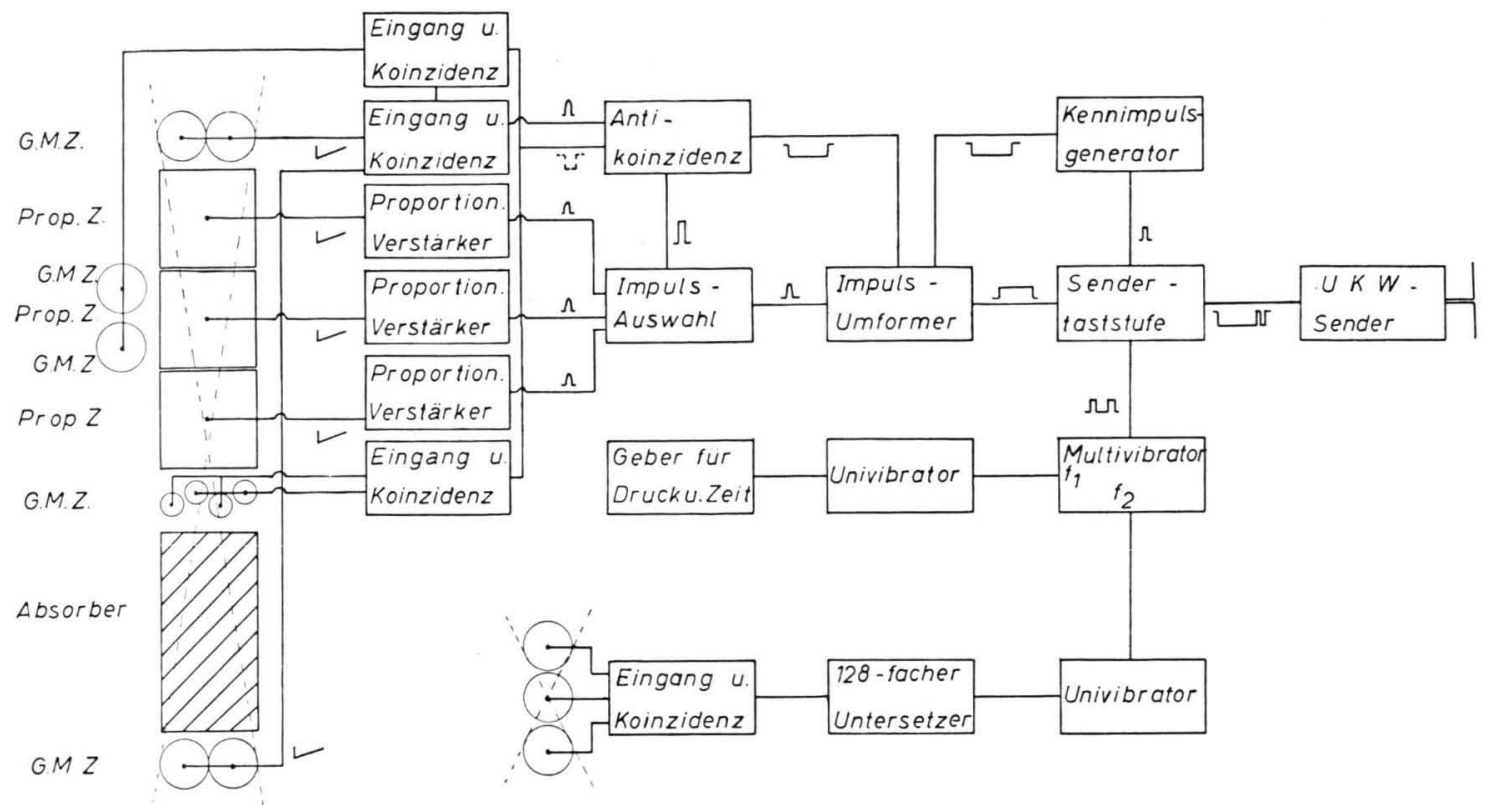

Abb. 1. Prinzipschaltbild der Ballonsonde.

an dieser Stelle nur eine kurze Darstellung an Hand eines Blockdiagramms gegeben (s. Abb. 1). Ein hochenergetisches Teilchen soll nur dann registriert werden, wenn es die drei Proportionalzähler und die den Einfallswinkel begrenzenden Geiger-Zähler durchdrungen hat. Dabei wird gleichzeitig eine bestimmte Mindestenergie durch den eingebauten Kupferabsorber von $72 \mathrm{~g} / \mathrm{cm}^{2}$ gefordert, so daß langsame Teilchen mit stark energieabhängiger erhöhter spezifischer Ionisation nicht gezählt werden können. Für die Unterdrückung von Schauern sorgt eine Lage von GEIGER-Zählern unterhalb der Proportionalzähler und solche seitlich von diesen über eine Antikoinzidenzschaltung. Der Gesamtabsorber beträgt (einschließlich der Zählerwände) $82,8 \mathrm{~g} / \mathrm{cm}^{2}$ äquivalent etwa $10,5 \mathrm{~cm}$ Blei; Protonen müssen eine kinetische Mindestenergie von $320 \mathrm{MeV}$ und $\mu$-Mesonen von $160 \mathrm{MeV}$ haben, um registriert zu werden.

Die geomagnetischen Abschneideenergien lagen bei allen Messungen bedeutend über diesen Grenzwerten. Bis zum untersten Proportionalzähler muß beim am 23. 8. 1960 verwendeten Gerät eine Schicht von 4,2 g pro $\mathrm{cm}^{2}$ Messing, $0,7 \mathrm{~g} / \mathrm{cm}^{2}$ Glas und $0,9 \mathrm{~g} / \mathrm{cm}^{2}$ Silicongummi, also insgesamt $5,8 \mathrm{~g} / \mathrm{cm}^{2}$ durchdrungen werden.

Die Geometriekonstante des Teleskops beträgt für die isotrope Richtungsverteilung $3,55 \mathrm{~cm}^{2}$ sterad und für die $\cos ^{2} \vartheta$-Abhängigkeit $3,42 \mathrm{~cm}^{2}$ sterad $(\vartheta$ ist der Einfallswinkel von der Vertikalen).

Wegen der starken Unsymmetrie der Ionisationsverteilung nach $\operatorname{LANDAU}^{2}$ wird die spezifische Ionisation durch Auswahl des kleinsten von drei Proportionalimpulsen gemessen (s. Abb. 2). Diese Impulse werden

2 L. Landau, J. Phys. (USSR) 8, 201 [1944]. vor der Auswahl getrennt verstärkt, geformt und dann gleichzeitig mit dem von den Geiger-Zählern kommenden ebenfalls geformten Impulsen stets maximaler Größe auf die Auswahlschaltung gegeben. Eine Schauerantikoinzidenz verhindert das Auftreten eines GeigerImpulses an dieser Stelle. Das resultierende Signal variabler Amplitude wird zur Funkübertragung in ein Signal variabler Dauer transformiert. Die Registrierung am Boden erfolgt über Oszillograph und Film.

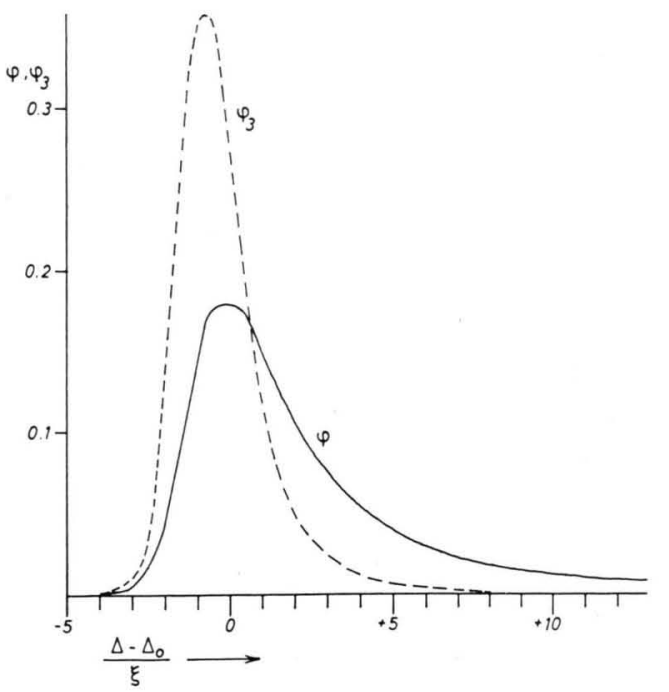

Abb. 2. Differentielle Ionisationsverteilung $\varphi$ nach LandaU und $\varphi_{3}$ nach Auswahl des kleinsten Ionisationsverlustes in drei getrennten Zählern. 


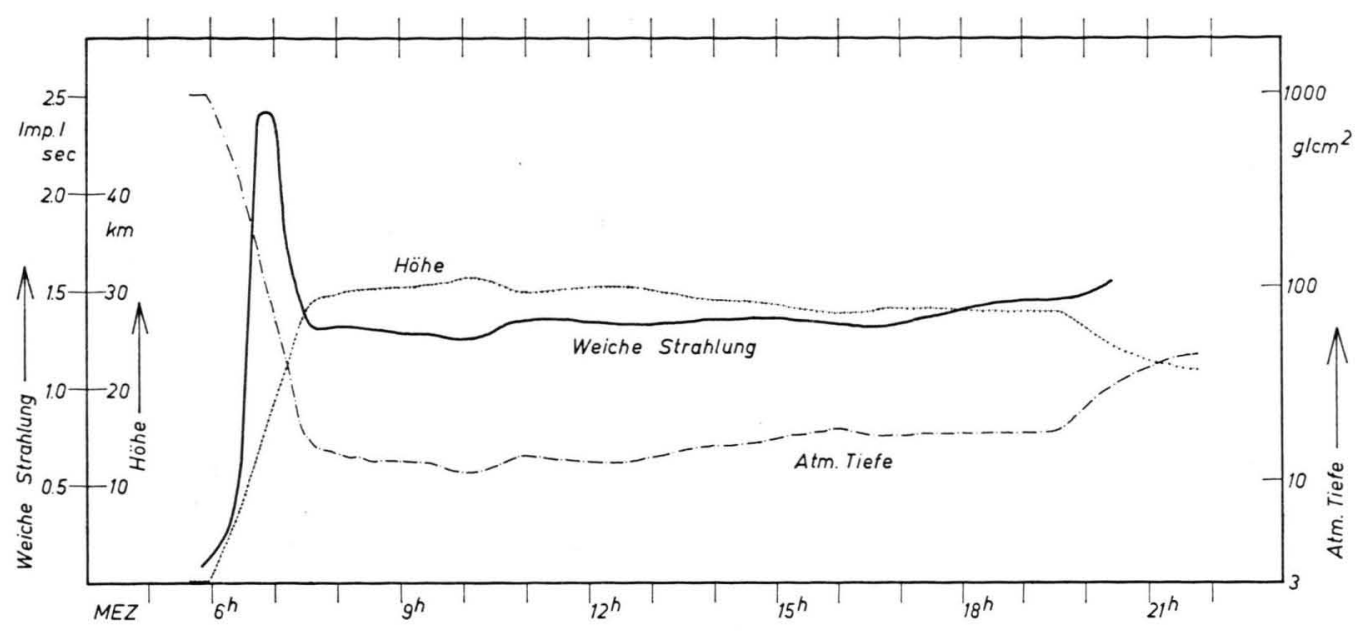

Abb. 3. Registrierungen von Druck, Höhe und weicher Strahlung am 23. 8. 1960.

Die weiche Strahlung wurde mit einem getrennten Teleskop aus Geiger-Zählern in Dreifach-Koinzidenz gemessen. Die Geometriekonstante beträgt für die isotrope Strahlung etwa $7,1 \mathrm{~cm}^{2}$ sterad und für die $\cos ^{2} \vartheta$. Abhängigkeit $5,9 \mathrm{~cm}^{2}$ sterad.

\section{Daten zum Ballonaufstieg vom 23. 8. 60}

Für diesen Aufstieg stand ein Plastikballon aus nicht dehnbarer Polyäthylenfolie zur Verfügung. Der Start fand am 23. 8. 1960 um 5.55 MEZ in Lindau/Harz (geogr. Breite $51^{\circ} 39^{\prime} \mathrm{N}$, geogr. Länge $10^{\circ} 07^{\prime} \mathrm{E}$ ) statt. Abb. 3 zeigt den während des Fluges registrierten Druck und die entsprechende Höhe als Funktion der Zeit. Das Volumen des Ballons betrug $3820 \mathrm{~m}^{3}$, das Eigengewicht 24,5 kg. Mit einem Bruttogewicht von $54 \mathrm{~kg}$ (Gewicht von Ballon und Nutzlast) war eine Höhe von etwa $31 \mathrm{~km}$ zu erwarten. Zusätzlich wurde beim Start ein Ballast von $3 \mathrm{~kg}$ festen Kohlendioxyds in einem Wärmeschutzkasten beigefügt, der bei einem eventuellen Überschießen des Ballons über die Gleichgewichtsdruckzone ein Absinken durch mäßige Ballastabgabe verhindern sollte, falls zuviel Gas abblasen sollte.

Die Proportionalimpulse konnten von 5.55 bis 18.45 Uhr registriert werden, danach waren die Batterien erschöpft. Die weiche Strahlung hingegen wurde fast bis $21 \mathrm{Uhr}$ gemessen.

\section{Meßergebnisse vom 23. 8. 60}

\section{a) Die Höhenabhängigkeit der harten Komponente}

Die während des raschen Aufstiegs registrierte Gesamtimpulshäufigkeit der harten Komponente wird in Abb. 4 in Abhängigkeit von der atmosphärischen Tiefe dargestellt. Die Ausbildung eines Maximums ist nicht zu erkennen. Wegen der kurzen
Meßzeiten sind die statistischen Fehler relativ groß, sie wurden in das Diagramm eingezeichnet. Der Bodenwert konnte jedoch recht genau über die Mesonenregistrierung in Lindau ermittelt werden, desgleichen ist der statistische Fehler der Teilchenflüsse beim Ausschwebedruck sehr klein.

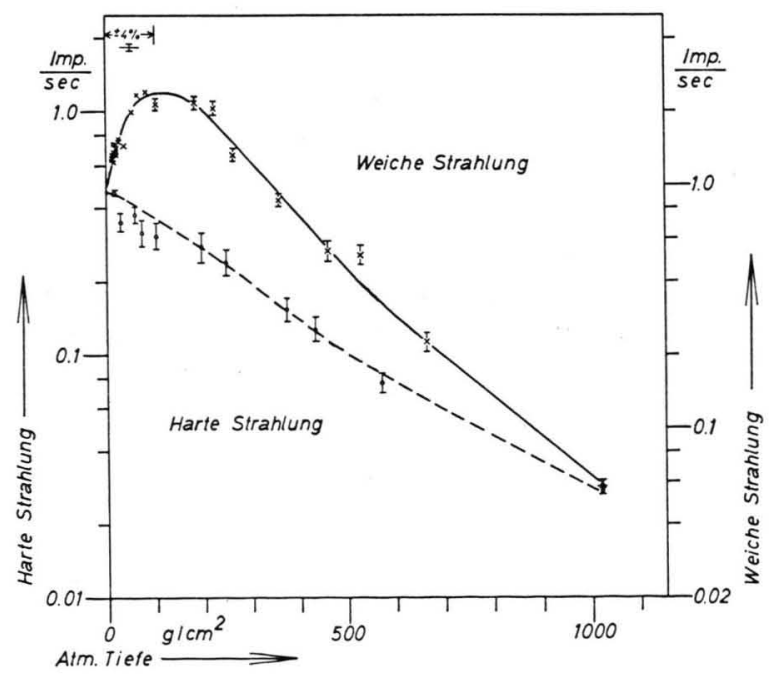

Abb. 4. Harte und weiche Strahlung als Funktion der atmosphärischen Tiefe.

\section{b) Die weiche Strahlung}

Die Höhenabhängigkeit der mit Dreifach-Koinzidenzen gemessenen weichen Strahlung zeigt Abb. 4 in Impulsen pro Sekunde; Fehler durch Totzeiten und zufällige Koinzidenzen wurden nicht berücksichtigt, sie bleiben stets unter $1 \%$. In Ausschwebehöhe 
wären $0,6 \%$, im Häufigkeitsmaximum $0,9 \%$ zu addieren. Der Verlauf der weichen Strahlungsintensität während des ganzen Fluges wurde in Abb. 3 neben Druck und Höhe als Funktion der Zeit aufgetragen.

Effekte durch zeitliche Schwankungen treten während des Aufstiegs praktisch nicht auf. Systematische Änderungen während der Ausschwebezeit sind durch Änderungen der atmosphärischen Tiefe bedingt.

Die Extrapolation auf $0 \mathrm{~g} / \mathrm{cm}^{2}$ der mit dem Dreifach-Koinzidenz-Teleskop gemessenen Gesamtstrahlung einschließlich der weichen Strahlung führt zu 0,141 Teilchen $/\left(\mathrm{cm}^{2} \mathrm{sec} \mathrm{sr}\right)$ und befindet sich damit in guter Übereinstimmung mit der extrapolierten harten Strahlung, wenn man den raschen Anstieg der Sekundärstrahlung mit der atmosphärischen Tiefe und die Albedo berücksichtigt.

\section{Die absoluten Teilchenintensitäten aus vertikaler Richtung}

Für die Gewinnung der auf $0 \mathrm{~g} / \mathrm{cm}^{2}$ extrapolierten Häufigkeiten der Gesamtstrahlung, der Protonen und $\alpha$-Teilchen wurden die Registrierungen während der Ausschwebephase von 7.30 bis $18.30 \mathrm{MEZ}$ verwendet. Bei den extrapolierten Werten werden obere und untere Grenzen angegeben, die sich auf die Berücksichtigung oder Vernachlässigung der Verluste beziehen, die in den oberen Teleskopschichten entstehen können. Es handelt sich hierbei um Kernreaktionen mit Auslösung interner Schauer, die durch den Schauerselektor zu einer Auslöschung des Ereignisses führen. Bei Schauern geringer Teilchenzahl und solchen mit sehr enger Bündelung kann

\begin{tabular}{|c|c|c|}
\hline & obere Grenze & untere Grenze \\
\hline $\begin{array}{l}\text { Gesa } \\
\text { Proto }\end{array}$ & $0,138 \pm 0,003$ & $0,130 \pm 0,003$ \\
\hline Beri & $0,118 \pm 0,003$ & $0,112 \pm 0,003$ \\
\hline $\begin{array}{l}\alpha \text {-Teilchen ohne } \\
\text { Korr. auf Kernauf- } \\
\text { spaltung } \\
\left(\Lambda=45 \mathrm{~g} / \mathrm{cm}^{2}\right)\end{array}$ & $0,0193 \pm 0,0006$ & $0,0173 \pm 0,0006$ \\
\hline $\begin{array}{l}\alpha \text {-Teilchen mit } \\
\text { Korr. auf } \\
\text { Kernaufspaltung }\end{array}$ & $0,0177 \pm 0,0006$ & $0,0162 \pm 0,0005$ \\
\hline
\end{tabular}

Tab. 1. Auf $0 \mathrm{~g} / \mathrm{cm}^{2}$ extrapolierte Werte vom 23. 8. 1960.

3 P. H. Fowler, R. R. Hillier u. C. J.Waddington, Phil. Mag. 2. 293 [1957].

4 E. Waibel, Nuovo Cim. Ser. X, 19, 482 [1961]. eine Registrierung erfolgen. Die tatsächlichen absoluten Häufigkeiten liegen demnach zwischen den beiden angegebenen Grenzen. Sie unterscheiden sich für die Protonen um $5 \%$, für die $\alpha$-Teilchen um $9 \%$.

Die angegebenen Fehler enthalten neben den mittleren statischen Fehlern 2,1\% für die Unsicherheit der Geometriekonstanten des Teleskops.

Die $\alpha$-Teilchen wurden einmal extrapoliert unter Zugrundelegung einer mittleren freien Weglänge von $45 \mathrm{~g} / \mathrm{cm}^{2}$ und einmal mit Berücksichtigung der Aufspaltung schwerer Kerne. Bei letzterem wurden die Werte für Spaltwahrscheinlichkeiten und freie Weglängen von Fowler, Hillier und Waddington ${ }^{3}$ verwendet.

\section{Die Schwankungen der primären Gesamt- strahlung und der $\alpha$-Teilchen}

Zur Ermittlung der Gesetzmäßigkeiten der Schwankungen werden vier Ballonaufstiege herangezogen, von denen einer in Weißenau und drei in Lindau/Harz gestartet wurden. Bei jeweils zwei Aufstiegen konnte das gleiche Teleskop verwendet werden (Gerät I am 24.4.57 und 16.7.59, Gerät II am 3.11.59 und 23.8.60). Über die drei Ergebnisse an den ersten drei Daten wurde bereits berichtet ${ }^{4}$. Zur Bestimmung der Schwankungen müssen die betreffenden Strahlungsintensitäten auf einen festen Ort umgerechnet werden. Dies ist notwendig, da Weißenau eine um ca. $3^{\circ}$ niedrigere geomagnetische Breite hat und bei den Flügen von Lindau aus weitere Abweichungen bis zu $1,5^{\circ}$ auftraten.

Die Korrekturfaktoren zwischen der unteren und oberen Grenze unterscheiden sich für beide Teleskope nur unwesentlich $(\leqq 0,5 \%)$. Die Extrapolation der $\alpha$-Teilchen auf $0 \mathrm{~g} / \mathrm{cm}^{2}$ mit Berücksichtigung der Aufspaltung der schweren Kerne wurde jedoch vereinheitlicht, indem die Häufigkeit der schweren Kerne mit einer Kernladung von $Z>2$ gleich $10 \%$ der $\alpha$-Teilchen-Intensität bei $0 \mathrm{~g} / \mathrm{cm}^{2}$ gesetzt wurde. Die Aufteilung in leichte, mittlere und schwere Kerne erfolgt mit $J_{\mathrm{L}}{ }^{0}=0,8 J_{\mathrm{M}}{ }^{0}$ und $J_{\mathrm{H}}{ }^{0}=0,4 J_{\mathrm{MI}}{ }^{0}$,

$$
J_{\mathrm{L}}{ }^{0}+J_{\mathrm{M}}{ }^{0}+J_{\mathrm{H}}{ }^{0}=0,10 J_{a}{ }^{0} \quad \text { (s. z. B. } \text { SinGER }^{5} \text { ). }
$$

Die Spaltwahrscheinlichkeiten wurden wieder aus ${ }^{3}$ entnommen; die Extrapolationsmethode wurde bereits ausführlich besprochen ${ }^{1}$.

\footnotetext{
5 S. F. Singer, Progr. Elementary Particle and Cosmic Ray Physics, North-Holland Publishing Co., Amsterdam 1958, Vol. IV, Chapt. IV.
} 
Zur genaueren Bestimmung der Intensitäten zwischen den oberen und unteren Grenzen ist eine $\mathrm{Ab}$ schätzung der Häufigkeit der Kernwechselwirkungen erforderlich, die tatsächlich zu einer Auslöschung durch die Schauerantikoinzidenz führen. Hierbei muß man folgendes kennen: a) die Geometrie der betreffenden GEIGER-Zähler, b) die Verteilung der Materieschichten im Proportionalteleskop, c) die Multiplizität der schnellen Teilchen nach der Reaktion, die die Materieschichten bis zu den Schauerzählrohren durchdringen können, also d) ihre Energieverteilung und e) ihre Winkelverteilung. Gewisse Schwierigkeiten bereiten die Punkte c bis e.

Aus den Untersuchungen von Sternen in Photoemulsionen (s. z. B. Anm. ${ }^{6}$ ) sind die Bezeichnungen „Schauerteilchen“, "graue Spuren“ und "schwere Spuren" bekannt. Bei letzteren handelt es sich im wesentlichen um Verdampfungsteilchen (Protonen, $\alpha$-Teilchen) mit kinetischen Energien vornehmlich unterhalb von $30 \mathrm{MeV}$. Wegen der Zählerwandstärken vom untersten Proportionalzähler bis in die Schauerzähler von ca. $1,5 \mathrm{~g} / \mathrm{cm}^{2}$ scheiden diese Teilchen zur Auslösung der Antikoinzidenz im wesentlichen aus. Die "grauen“ Teilchen (Protonen, $\pi$-Mesonen) haben mittlere Energien von etwa $120 \mathrm{MeV}^{6}$ und bewirken mit den relativistischen „Schauerteilchen" großer Reichweite die Schauerunterdrückung. Für die Durchdringung des Teleskops bis zu den Zählern $\mathrm{S}_{1}$ (s. Abb. 5) ist für Protonen eine kinetische Energie von ca. $70 \mathrm{MeV}$, für $\pi$-Mesonen von ca. $35 \mathrm{MeV}$ erforderlich.

Wegen der Winkelverteilungen wurde die $\mathrm{Ab}$ schätzung der Korrektur für drei Abstände (den Schichten zwischen und über den Proportionalzählern) von den unteren Antikoinzidenzzählern vorgenommen; außerdem wurde die Multiplizität in Abhängigkeit von der Primärenergie berücksichtigt.

Im Falle der primären $\alpha$-Teilchen ist die Aufspaltung des Teilchens in seine i. allg. vier Nukleonen bei jedem Kernstoß sehr wahrscheinlich, weil die kinetische Energie wesentlich höher ist als die Bindungsgenergie. Die Winkelverteilung im Laborsystem läßt sich mit der Annahme einer isotropen Richtungsverteilung der Nukleonen im Schwerpunktsystem nach dem Auseinanderplatzen berechnen. Mit zunehmender Energie wird der Kegel der Nukleonenspuren sehr eng, aber die Zahl der Sekundärteilchen wächst dafür rasch. Wegen der Zahl der beteiligten

6 W. Heisenberg, Kosmische Strahlung, Springer-Verlag, Berlin 1953, Kap. II, S. 69 ff.

geladenen Teilchen von mindestens zwei ist der Wirkungsgrad der Antikoinzidenz recht gut. Es ergibt sich, daß bei $\alpha$-Teilchen nur $5-10 \%$ der Kernreaktionen nicht ausgelöscht werden. Das sind hauptsächlich die Fälle im unteren Energiebereich oberhalb der geomagnetischen Abschneideenergien ( $>0,6 \mathrm{GeV} /$ Nukleon). Diese verursachen im Ionisationsspektrum kaum Störungen, da sie nur rund $1 \%$ der gesamten $\alpha$-Häufigkeit ausmachen und in den meisten Fällen die Ionisation dieser Teilchenschauer in den $\alpha$-Bereich selbst fällt.

Abb. 5. Skizze zur Wirksamkeit der Schauerunterdrükkung. Näheres im Text.

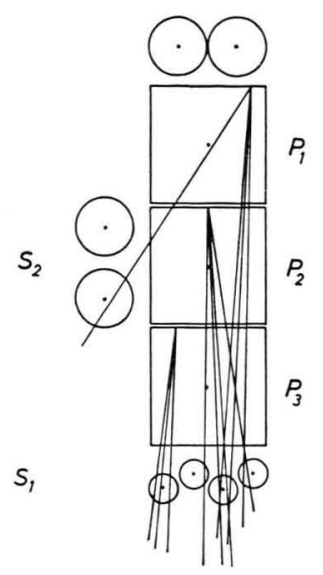

Bei den primären Protonen ist der Antikoinzidenzwirkungsgrad bei den entsprechenden kinetischen Energien pro Nukleon wesentlich geringer. Allerdings liegt die untere Grenze der Energie - die geomagnetische Abschneideenergie - bereits oberhalb von 1,5 GeV. Eine entsprechende Abschätzung der Antikoinzidenzrate führt zu einer Löschung der Ereignisse mit Kernwechselwirkungen im Teleskopmaterial von nur ca. $50 \%$. Das besagt, daß etwa $3 \%$ der gesamten Protonenhäufigkeit unterdrückt werden.

Die Korrekturfaktoren, die an den unteren Grenzwerten der Häufigkeiten anzubringen sind, betragen damit für die Protonen 1,03 und für die $\alpha$-Teilchen 1,08 .

In der Tab. 2 wurden die mit diesen Faktoren korrigierten Werte eingesetzt für die $\alpha$-TeilchenKomponente mit Berücksichtigung der Kernaufspaltung in der Atmosphäre. Außerdem werden die auf Lindau reduzierten Häufigkeiten der primären $\mathrm{Ge}$ samtstrahlung und der $\alpha$-Teilchen, sowie die zugehörigen Bodenwerte (für Lindau) angegeben. Die beigefügten mittleren Fehlergrenzen enthalten die statistisischen Fehler und die Unsicherheit der Teleskopfaktoren. 


\begin{tabular}{|c|c|c|c|c|c|c|c|c|}
\hline & $\begin{array}{l}\text { Gesamt- } \\
\text { strahlung } \\
\text { Teilchen } \\
\text { pro } \mathrm{cm}^{2} \mathrm{~s} s r \\
\text { direkt }\end{array}$ & $\begin{array}{c}\alpha \text {-Teilchen } \\
\text { pro } \mathrm{cm}^{2} \mathrm{~s} \text { sr } \\
\text { direkt }\end{array}$ & $\begin{array}{l}\text { Bodenwert } \\
\text { der Sonde } \\
\text { direkt } \\
\mathrm{Imp} / \mathrm{s}\end{array}$ & $\begin{array}{c}\text { Gesamt- } \\
\text { strahlung } \\
\text { Umrechnung } \\
\text { auf Lindau }\end{array}$ & $\begin{array}{c}\alpha \text {-Teilchen } \\
\text { Umrechnung } \\
\text { auf Lindau }\end{array}$ & $\begin{array}{c}\text { Neu- } \\
\text { tronen } \\
\text { Lin- } \\
\text { dau*) }\end{array}$ & $\begin{array}{c}\text { geomag- } \\
\text { netische } \\
\text { Mindest- } \\
\text { steifig- } \\
\text { keit } \\
\text { in GV }\end{array}$ & $\begin{array}{l}\text { Teleskop } \\
c(0) \mathrm{cm}^{2} \mathrm{sr}\end{array}$ \\
\hline 24. 4.57 & $0,093 \sqsupset$ & 0,0114 & $0,0242=$ & $0,110 \pm$ & $0,0132=$ & 518 & 3,08 & $2,96 \pm$ \\
\hline 16. 7.59 & $0,087 \pm 0,003$ & $0,0116 \pm 0,0008$ & $0,0212 \pm 0,0005$ & $0,084 \pm 0,002$ & $0,0113 \pm 0,0008$ & 45840 & 2,20 & $2,96 \pm 0,075$ \\
\hline 3.11 .59 & $0,101 \pm 0,005$ & $0,0130 \pm 0,0026$ & $0,0272 \pm 0,0005$ & $0,104 \pm 0,005$ & $0,0134 \pm 0,0026$ & 54550 & 2,49 & $3,55 \pm 0,075$ \\
\hline 23. 8.60 & $0,134 \pm 0,003$ & $0,0175 \pm 0,0006$ & $0,0270 \pm 0,0005$ & $0,134 \pm 0,003$ & $0,0175 \pm 0,0006$ & 55220 & 2,38 & $3,55 \pm 0,075$ \\
\hline
\end{tabular}

Tab. 2. Teilchenflüsse bei $0 \mathrm{~g} / \mathrm{cm}^{2}$. * Auf konstanten Druck reduziert.

Die Reduktion auf die geomagnetische Breite von Lindau wurde über die integralen Impulsspektren der Komponenten in Abhängigkeit vom geomagnetischen Abschneideimpuls des exzentrischen Dipolfeldes gewonnen, der über Deutschland recht gut mit den Werten nach Quenby und $W_{\text {ebber }}{ }^{7}$ übereinstimmt. Zur Berücksichtigung der zeitlichen Schwankungen durch die Sonnenaktivität wurden die für eine elektrische Modulation von EHMERT ${ }^{8}$ berechneten und an die experimentellen Spektren von McDonald ${ }^{9}$ angeschlossenen Kurven verwendet.

Zur Bestimmung der relativen Schwankungen der primären Strahlung gegenüber der Strahlung am Boden wurden in Abb. 6 die auf Lindau/Harz umgerechneten Häufigkeiten der Gesamtstrahlung und der $\alpha$-Teilchen in logarithmischem Maßstab über dem Logarithmus der in Lindau registrierten Neutronen aufgetragen. Der logarithmische Schwankungsfaktor für die primäre Gesamtstrahlung beträgt im Mittel in bezug auf die Neutronen am Boden $2,1 \pm 0,3$ und für die primären $\alpha$-Teilchen ebenfalls $2.1 \pm 0.3$.

Die integrale Häufigkeit der $\alpha$-Teilchen bei $0 \mathrm{~g}$ pro $\mathrm{cm}^{2}$ beträgt im Mittel $13 \%$ der Intensität der Gesamtstrahlung und das Verhältnis der Intensitäten von Protonen und $\alpha$-Teilchen liegt bei 6,6 bei gleicher magnetischer Steifigkeit.

Unsere Ergebnisse sind damit in guter Übereinstimmung mit denen von McDonald und Webber ${ }^{10}$ und von MEyer ${ }^{11}$.

Die Übereinstimmung der Schwankungen der Intensitäten von Protonen bzw. der primären Gesamtstrahlung und der $\alpha$-Teilchen während der ForbushEffekte und die gleiche Abhängigkeit im Bereich der

7 J. J. Quenby u. W. R. Webber, Phil. Mag. 4, 90 [1959].

8 A. Енmert, Proc. Moscow Cosmic Ray Conf. 1959, 4, 142 [1960]. - A. Ehmert, Physikertagung Wiesbaden Hauptvorträge, Physik-Verlag, Mosbach 1961, S. 13. A. Ehmert, Space Research, Proc. I. Int. Space Science

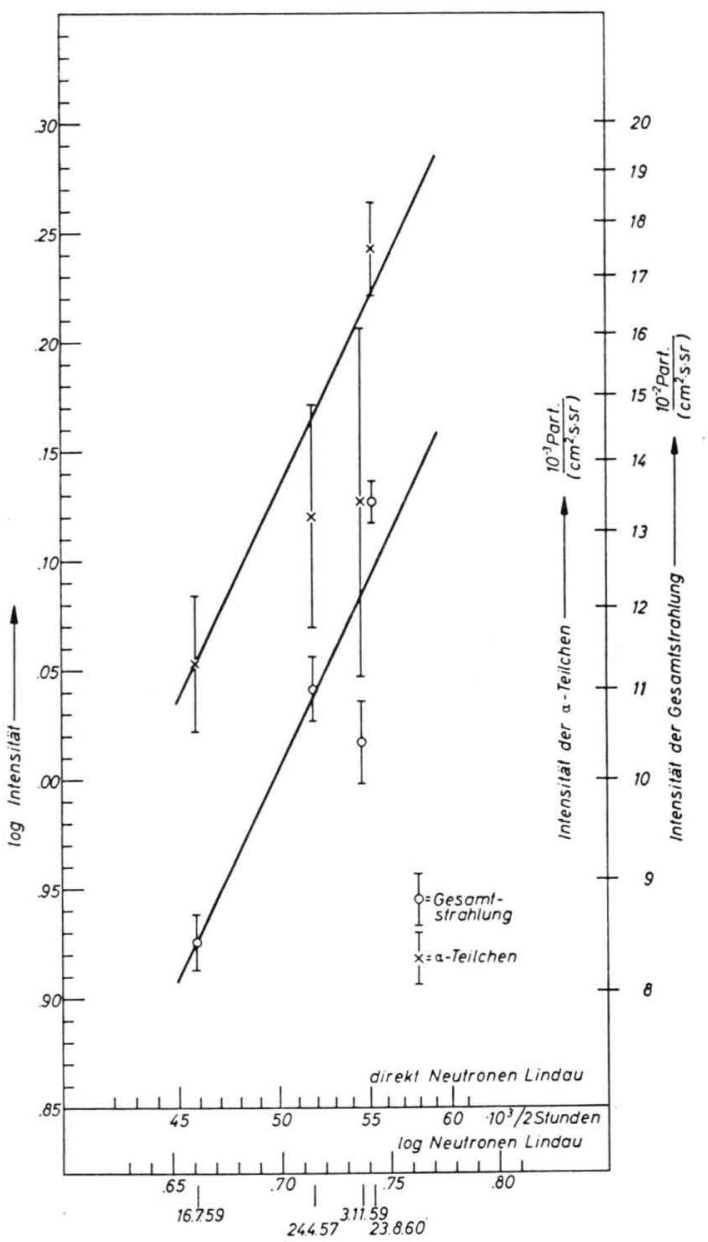

Abb. 6. Integrale extrapolierte Häufigkeiten der $\alpha$-Teilchen und der Gesamtstrahlung von verschiedenen Ballonaufstiegen aufgetragen über den Werten der Neutronenregistrierungen am Boden in Lindau. Beide Maßstäbe haben logarithmische

Teilungen zur Ermittlung der Schwankungsfaktoren.

Symp., Amsterdam 1960, S. 1000.

9 F. B. McDoxald, Phys. Rev. 109, 1367 [1958].

10 F. B. McDonald u. W. R. Webber, Space Research, Proc.

I. Int. Space Science Symp., Amsterdam 1960, S. 968.

11 P. Meyer, J. Geophys. Res. 65, 3881 [1960]. 
Intensitätsänderungen im Sonnenzyklus weisen auf einen gleichartigen Modulationsmechanismus für beide Vorgänge hin ${ }^{8}$. Da die gemessenen Relationen der integralen Häufigkeiten zwischen Protonen und $\alpha$-Teilchen jeweils bei gleichen geomagnetischen Mindeststeifigkeiten bestehen, liegt es nahe, die Ursache der Modulationen in magnetischen Feldern zu suchen, doch führt auch die elektrische Modulation zu einer Deutung.

Verschiedene Mechanismen auf magnetischer Basis wurden vorgeschlagen; so die Ablenkung der geladenen Teilchen an Plasmaströmen (DormanN ${ }^{12}$, AlfvÉN ${ }^{13}$ ) ungeordneten im Plasma gebundenen Magnetfeldern (Morrison ${ }^{14}$, PARker ${ }^{15}$ ) und von der Sonne durch Plasma mitgeführte, jedoch mit ihr noch verbundene magnetische Felder z. Tl. als magnetische „Flaschen“, die erst nach Passieren des Erdkreises turbulent werden (Piddington ${ }^{16}$, Gold ${ }^{17}$, McCracken und Palmeira ${ }^{18}$ ). Letztere bieten eine sehr gute Deutungsmöglichkeit bei der Heranführung von niederenergetischer solarer Ultrastrahlung zur Erde wie z. B. im November 1960, wo insbesondere nach Beginn des geomagnetischen Sturmes erhöhte Strahlung mit Neutronenmonitoren in mittleren und höheren Breiten nachgewiesen werden konnte. Insbesondere kann der zeitliche Verlauf der Ereignisse bei chromosphärischen Eruptionen verstanden werden.

Bei den meisten dieser Theorien stellt man sich die Intensitätsverminderung der kosmischen Strahlung während der Forbush-Effekte als Reflexion bzw. Rückstreuung der Teilchen an den Magnetfeldern, der von der Sonne ausgestoßenen Plasmawolken vor. Die Modulation des Steifigkeitsspektrums könnte z. B. dadurch erfolgen, daß eine bestimmte Häufigkeitsverteilung der Wolkengrößen mit inhomogenen Feldern vorliegt. Die niederenergetischen Teilchen, also allgemein solche mit geringer magnetischer Steifigkeit $p c /(z e)$, würden starke und häufige Rückstreuungen bzw. eine erschwerte Diffusion zur Erde haben gegenüber den hochenergetischen. Es müßten jeweils relativ viele kleine und wenige große Wolken mit magnetischen Inhomogenitäten vorhanden sein, die sich etwa innerhalb

12 L. L. Dorman, Cosmic Ray Variations, State Publishing House for Technical and Theoretical Literature, Moscow 1957.

13 H. Alfvén, Cosmical Electrodynamics, Clarendon Press, Oxford 1950.

14 P. Morrison, Phys. Rev. 101, 1397 [1956].

15 E. N. Parker, Phys. Rev. 110, 1445 [1958]. konzentrischer Kugelschalen von der Sonne entfernten und ausdehnten. Im Abstand Erde-Sonne würde man bei Wolkendurchmessern um eine Größenordnung größer als der Krümmungsradius der Teilchen zu Feldstärken von $10^{-4}$ bis $10^{-3} \mathrm{Gau} ß$ kommen für Protonenenergien von $10^{9}$ bis $10^{10} \mathrm{eV}$. Bei größeren Wolken sind entsprechend schwächere Felder erforderlich. Die langsame Erholung nach einem Forbush-Effekt und die stufenweise Abnahme der Intensität im Sonnenzyklus bei erhöhter Aktivität mit sehr langsamem Wiederanstieg wäre durch die Expansion der Kugelschalen mit Plasmawolken mit abnehmendem „Reflexionsfaktor“ bzw. erneuter Erzeugung derartiger Wolkenfronten zu deuten. Neben diesen Reflexionen und Streuungen ohne wesentliche Energieabgabe können nach Singer ${ }^{19}$ Abbremsungen als umgekehrte Fermi-Prozesse an diesen Feldinhomogenitäten auftreten. Dies gilt insbesondere für den niederenergetischen Teil des Spektrums.

Ein scharfer Einsatz eines Forbush-Effektes wird verursacht, wenn die Erde in eine Plasmawolke gerät. Die mehr oder weniger starke Trennung der Wolken verhindert die Ausbildung eines scharfen cut-offs.

Andere magnetische Modulationsvorgänge kommen insbesondere in Sonnennähe in Betracht, da dort größere zusammenhängende geordnete Felder vorkommen können. Doch liegen nur wenige qualitative Berechnungen über die magnetischen Modulationsmechanismen vor.

Daneben wurde die Abbremsung der kosmischen Strahlung durch elektrische Felder untersucht und die Spektren berechnet (Fonger ${ }^{20}$, EhMert ${ }^{8}$ ).

Die gefundenen experimentellen integralen Intensitäten passen sehr gut zu den von EHMERT berechneten Spektren, die an Messungen von McDonald angeschlossen wurden. Nimmt man für die differentiellen Spektren die gleichen experimentellen Relationen zwischen Protonen und $\alpha$-Teilchen an - was von MCDonald direkt gemessen werden konnte - , so ergibt sich, daß mit den bisherigen Berechnungen auf magnetischer Modulationsbasis noch keine volle Übereinstimmung der Spektralformen erzielt werden konnte. Den Abweichungen, die bei den differen-

16 J. H. Piddington, Phys. Rev. 112, 589 [1958].

17 T. Gold, J. Geophys. Res. 64, 1665 [1959].

18 K. G. McCracken u. R. A. R. Palmeira, J. Geophys. Res. 65, 2673 [1960].

19 S. F. Singer, Nuovo Cim. Suppl. (10) 8, 342 [1958].

20 W. H. Fonger, Phys. Rev. 91, 351 [1953]. 
tiellen Spektren für die elektrische Modulation bei sehr niedrigen Teilchenenergien blieben, stehen die Schwierigkeiten der Meßtechnik gegenüber. Die quantitative Übereinstimmung der Spektren ist bis jetzt bei diesem Modulationstyp am besten, wenn auch noch nicht genügend über die Entstehung der erforderlichen elektrischen Bremsung bekannt ist.

In diesem Zusammenhang muß auf die Messungen von Appa $\mathrm{RaO}^{21}$ und Überlegungen von Appa RAO und KAPLON ${ }^{22}$ hingewiesen werden, wonach der Anteil des ${ }^{3} \mathrm{He}$-Isotops der kosmischen Strahlung ca. $35 \%$ der Gesamthäufigkeit der He-Kerne ausmachen soll und eine experimentelle Bestimmung der differentiellen Impulsspektren eine erhebliche Beeinflussung erfährt, wenn mit einheitlichen ${ }^{4} \mathrm{He}$ Kernen gerechnet wird. Ähnliches gilt für die Isotopenzusammensetzung der einfach geladenen Teilchen ebenfalls ausgeprägt im Bereich der geomagnetischen Abschneideimpulse. Mit höheren Energien wird diese Verfälschung auf experimenteller Basis

21 M. V. K. Appa Rao, Phys. Rev. 123, 295 [1961]. bei Nichtbeachtung der Isotopenanteile vernachlässigbar.

Zur weiteren Klärung und Entscheidung ergibt sich daher die Forderung nach Messungen der integralen und differentiellen Spektren, die bis zu möglichst niedrigen Energien reichen, also in hohen geomagnetischen Breiten ausgeführt werden müßten, sowie weitere Untersuchungen über die Isotopenanteile für die primären Komponenten mit ein- und zweifacher Kernladung. Diesen Experimenten kommt eine erhöhte Bedeutung zu für Zeiten unmittelbar am Beginn eines Forbush-Effektes; außerdem ist ihr Wert für die Messung solarer Zusatzstrahlung angezeigt. Andererseits muß versucht werden, die modifizierten magnetischen Modulationsmechanismen quantitativ zu fassen.

Herrn Prof. Dr. A. Ehmert und Herrn Dr. G. Pfotzer danke ich für förderliche Diskussionen und der Deutschen Forschungsgemeinschaft für eine Sachbeihilfe auf Antrag von Herrn Prof. Ehmert.

22 M. V. K. Appa Rao u. M. F. Kaplon, Nuovo Cim. Ser. X, 21, 369 [1961].

\title{
Isotopenüberführung, äußere Überführung und Selbstdiffusion von $\mathrm{Li}$ in festem $\mathrm{Li}_{2} \mathrm{SO}_{4}$ bei $600-790^{\circ} \mathrm{C}$
}

\author{
Von Arnold Lundén \\ Aus dem Physikalischen Institut der Chalmers Technischen Hochschule, Göteborg \\ (Z. Naturforschg. 17 a, 142-146 [1962]; eingegangen am 29. November 1961)
}

\begin{abstract}
Festes $\mathrm{Li}_{2} \mathrm{SO}_{4}$ wurde mit einer Kathode aus flüssigem $\mathrm{Zn}$ und einer Anode aus Au-Pt elektrolysiert. ${ }^{7} \mathrm{Li}$ wurde an der Anode angereichert. Der Masseneffekt (relative Differenz der Wanderungsgeschwindigkeiten/relative Differenz der Massen) ändert sich praktisch nicht mit der Temperatur. Er ist $\mu=-0,14$, d. h. ebenso groß wie in geschmolzenen Halogeniden. Der Selbstdiffusionskoeffizient des Kations wurde aus der praktischen Reichweite der Isotopentrennung geschätzt:

$$
D^{+}=0,015 \exp (-10500 / R T) \text {. }
$$

Dabei ist $D^{+}$in $\mathrm{cm}^{2} \mathrm{sec}^{-1}$ und $R$ in $\mathrm{cal} \mathrm{Mol}^{-1} \operatorname{grad}^{-1}$ gemessen. Die erhaltene Aktivierungsenergie ist zwar größer als in geschmolzenen Salzen, aber der Unterschied ist nicht signifikant. Aus der Verschiebung der Anode wurde die Überführungszahl des Kations gemessen: $U^{+}=1,00$. Es wurde festgestellt, daß das Faradaysche Gesetz im festen $\mathrm{Li}_{2} \mathrm{SO}_{4}$ gilt.

Einige Fragen zur eventuellen Anwendung von $\mathrm{Li}_{2} \mathrm{SO}_{4}$ für die Anreicherung von Isotopen in großen Mengen werden diskutiert.
\end{abstract}

Im Temperaturbereich von $575{ }^{\circ} \mathrm{C}$ bis zum Schmelzpunkt (etwa $850{ }^{\circ} \mathrm{C}$ ) bildet Lithiumsulfat eine kubische Modifikation, in der die LithiumIonen eine große Beweglichkeit haben ${ }^{1}$. Die elektrische Leitfähigkeit ist etwa gleich groß wie in Schmel-

1 T. Förland u. J. Krogh-Moe, Acta Chem. Scand. 11, 565 [1957]; Acta Cryst. 11, 224 [1958].

2 A. Benrath u. K. Drekopf, Z. phys. Chem. 99, 57 [1921]. zen ${ }^{2,3}$ und ihre Aktivierungsenergie ist ungewöhnlich klein ${ }^{3}$. Der Isotopieeffekt bei der elektrolytischen Überführung der Lithium-Ionen und ihr Selbstdiffusionskoeffizient sind auch von derselben Größenordnung wie in Schmelzen ${ }^{4}$. Diese beiden

3 T. Förland u. J. Krogh-Moe, private Mitteilung.

4 A. LundÉs, Z. Naturforschg. 15 a, 365 [1960]. 